\title{
Sarcomatoid Hepatocellular Carcinoma with Spontaneous Intraperitoneal Bleeding
}

\author{
Yukinobu Watanabe ${ }^{1}$, Naoki Matsumoto ${ }^{1}$, Masahiro Ogawa ${ }^{1}$, \\ Mitsuhiko Moriyama ${ }^{1}$ and Masahiko Sugitani ${ }^{2}$
}

\begin{abstract}
An 80-year-old woman was admitted to our hospital with right hypochondralgia. Abdominal ultrasonography showed a well-circumscribed round lesion in segments 5 and 6 of the liver. Contrast-enhanced ultrasonography (CEUS) indicated that the tumor was enhanced in the arterial phase, changing to hypo-enhanced 30 seconds following contrast injection. After admission, the patient complained of epigastralgia, and CEUS subsequently demonstrated extravasation from the tumor. Although abdominal angiography was successful for achieving hemostasis, the tumor grew rapidly and the patient died. An autopsy revealed the presence of sarcomatoid hepatocellular carcinoma (HCC). The literature is lacking in details regarding CEUS findings for sarcomatoid HCC and rupture. We herein report a rare case of sarcomatoid HCC.
\end{abstract}

Key words: sarcomatoid hepatocellular carcinoma, rupture, contrast-enhanced ultrasonography, Sonazoid

(Intern Med 54: 1613-1617, 2015)

(DOI: 10.2169/internalmedicine.54.3523)

\section{Introduction}

Sarcomatoid hepatocellular carcinoma (HCC) progresses rapidly, with widespread metastasis resulting in a very poor prognosis (1). However, the findings of contrast-enhanced ultrasonography (CEUS) in such patients have yet to be reported, and intraperitoneal bleeding of sarcomatoid HCC is rare. We herein report a case of sarcomatoid HCC with intraperitoneal hemorrhage and discuss the associated CEUS findings in detail.

\section{Case Report}

An 80-year-old woman was admitted to our hospital with right hypochondralgia and anemia. The patient had a history of a giant liver cyst that had been treated with percutaneous liver abscess drainage and ethanol injection; however, she had no past history of alcohol overuse.

Upon admission, the patient was $139 \mathrm{~cm}$ tall and weighed $48 \mathrm{~kg}$, with a body mass index (BMI) of 24.8. A physical examination showed pale palpebral conjunctiva, and the liver was not palpable. Laboratory tests revealed a decreased level of hemoglobin $(8.9 \mathrm{mg} / \mathrm{dL})$ and an elevated concentration of C-reactive protein $(2.9 \mathrm{mg} / \mathrm{dL})$. Negative results were obtained for both hepatitis B surface antigens and hepatitis $\mathrm{C}$ virus antibodies. The levels of serum tumor markers were within the normal limits.

Abdominal ultrasonography showed a well-circumscribed round lesion exhibiting heterogeneous internal echogenicity in segments 5 and 6 of the liver. The lesion contained a central anechoic area (Fig. 1A). A cystic lesion was apparent in segments 6-7 of the right lobe, touching the tumor and measuring $62 \mathrm{~mm}$ in diameter. Doppler ultrasonography revealed prominent hepatic arteries encircling the lesion. No blood flow signals were detected inside the lesion (Fig. 1B).

On the dorsal part of the lesion, branching vessels were enhanced in the arterial phase on CEUS with the contrast agent Sonazoid (Daiichi-Sankyo, Tokyo, Japan) using the GE LOGIQ 7 ultrasound system (GE Healthcare, Milwaukee, USA). This imaging examination also showed a contrast effect arising from the central area of the lesion (Fig. 1C). Gradually, almost all dorsal regions showed enhancement (Fig. 1D), and a change to hypo-enhancement in

${ }^{1}$ Department of Gastroenterology and Hepatology, Nihon University School of Medicine, Japan and ${ }^{2}$ Department of Pathology, Nihon University School of Medicine, Japan

Received for publication July 22, 2014; Accepted for publication October 14, 2014

Correspondence to Dr. Naoki Matsumoto, matsumotosg@yahoo.co.jp and koushin0809@tigers-net.com 

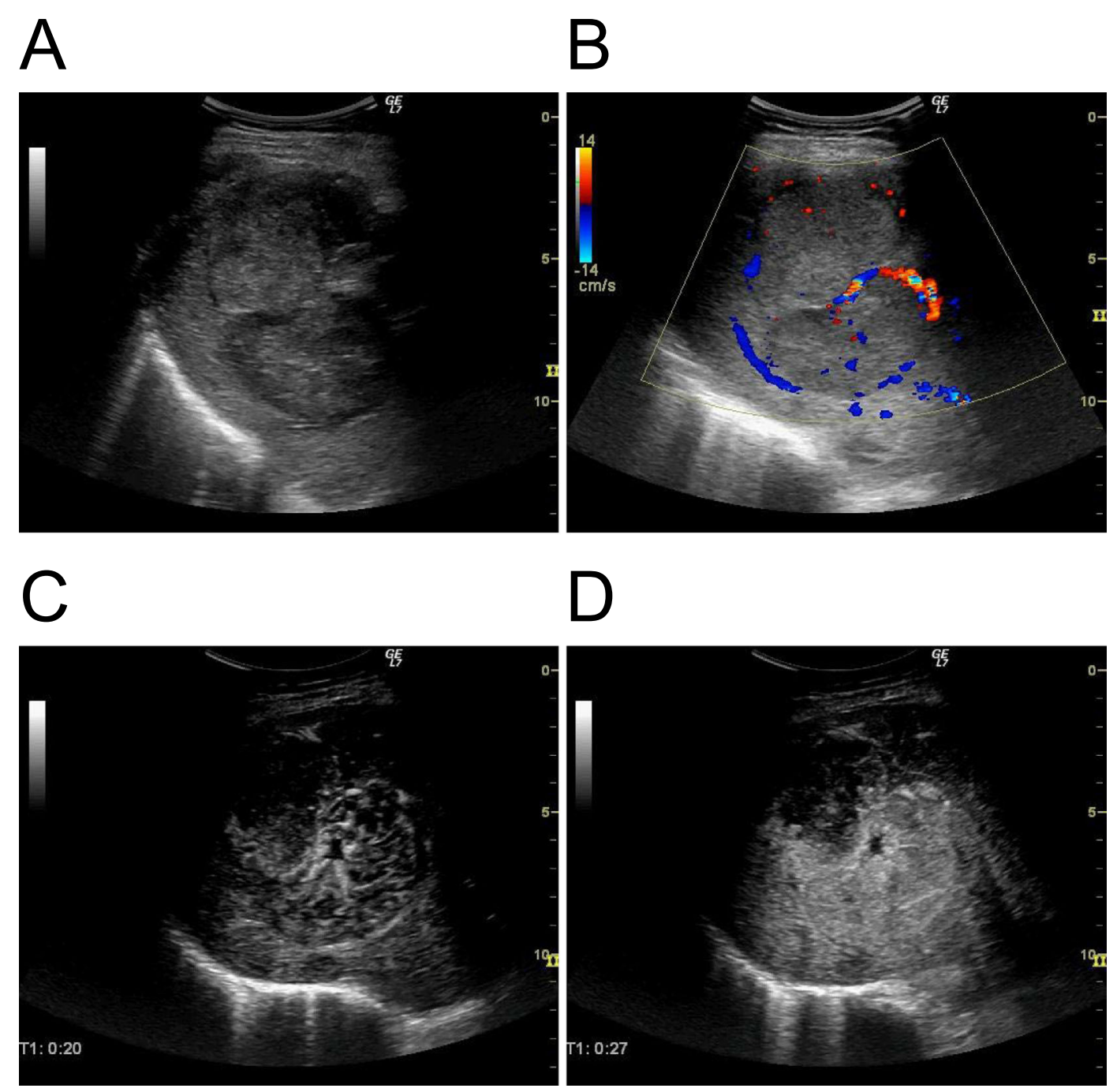

Figure 1. Ultrasonography (A) demonstrated a mixture of high- and low-echoic signs in a lesion containing an anechoic area in S6 to S5. Doppler ultrasonography (B) demonstrated prominent hepatic arteries that encircled the lesion. No blood flow signals were detected inside the lesion. Contrastenhanced ultrasonography (CEUS) using Sonazoid in the arterial phase (C, D) disclosed enhancement of the dorsal part of the tumor. The ventral lesion exhibited a contrast effect only along the periphery.

these areas began at 30 seconds after contrast injection; the peripheral area of the lesion remained enhanced. In addition, the ventral part of the lesion showed ring enhancement in the arterial phase, while the interior remained unenhanced. Eight minutes later, almost all regions of the lesion returned to a hypo-enhanced state.

A dynamic study of computed tomography (CT) scanning of the abdomen demonstrated an irregularly shaped hypodense tumor in S5 and S6, with peripheral ring-like enhancement during the arterial and portal phases (Fig. 2).

After admission, the patient complained of epigastralgia, and laboratory tests revealed a decreased hemoglobin level. CEUS was again performed to assess the tumor in S7, the results of which revealed high-intensity echoes of Sonazoid microbubbles in the ascites surrounding the tumor (Fig. 3).

Emergency abdominal angiography showed a circumscribed non-vascular lesion with a combination of tumor and cystic features; however, there was no clear evidence of ex- travasation (Fig. 4). Four coils were placed in the right hepatic artery, and homeostasis was successfully achieved. However, the tumor grew rapidly, and the patient died 57 days after admission.

An autopsy was performed, which revealed a necrotic tumor in the right lobe of the liver exposed on the lower surface of the liver in the abdominal cavity and measuring $12 x$ $9 \times 12 \mathrm{~cm}$ (Fig. 5). Microscopically, the tumor tissue displayed proliferation of spindle-shaped cells with irregularly shaped nuclei. There were no fibrotic changes in the nontumorous areas of the liver. An immunohistochemical examination demonstrated positive results for only vimentin (Dako, Carpinteria, USA) and hepatocytes (Dako) (Fig. 6). We diagnosed the patient with sarcomatous HCC based on the funicular structure with sinusoid-like veins and positive findings for immunohistochemistry using hepatocyte-specific Hepatocytes antibodies in the necrotic lesion. 

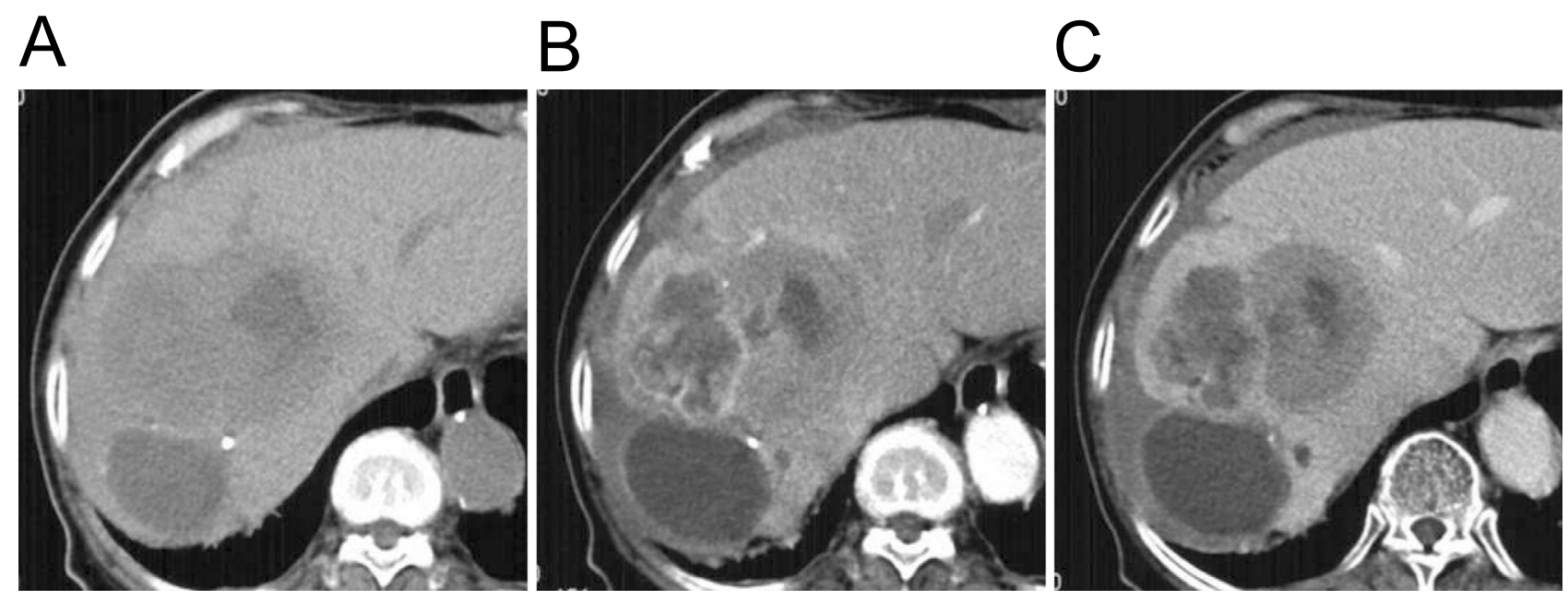

Figure 2. Plane computed tomography (CT) (A) demonstrated an irregularly shaped hypodense tumor with a water density area in S5 and S6. There was a cystic lesion, touching the tumor, in S6. The tumor displayed peripheral ring-like enhancement in the arterial and portal phases $(\mathrm{B}, \mathrm{C})$.

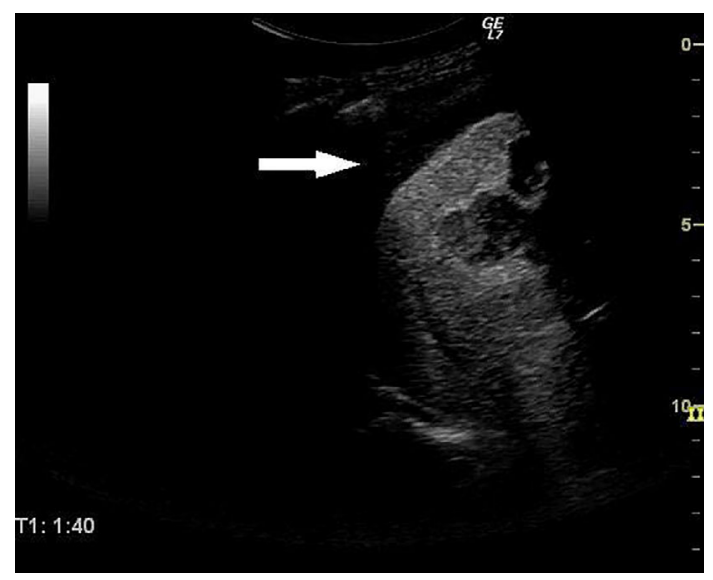

Figure 3. Punctate high-intensity echoes of Sonazoid microbubbles were observed in the ascites on CEUS.

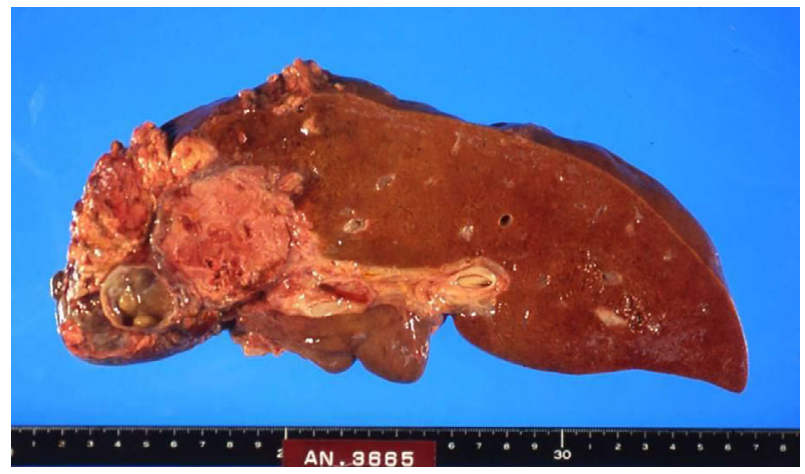

Figure 5. The macroscopic findings showed a necrotic tumor in the right lobe of the liver, exposed on the lower surface of the liver in the abdominal cavity and measuring $12 \times 9 \times 12 \mathrm{~cm}$. There was also a cystic lesion in S6 that touched the tumor, measuring $5 \mathrm{~cm}$.

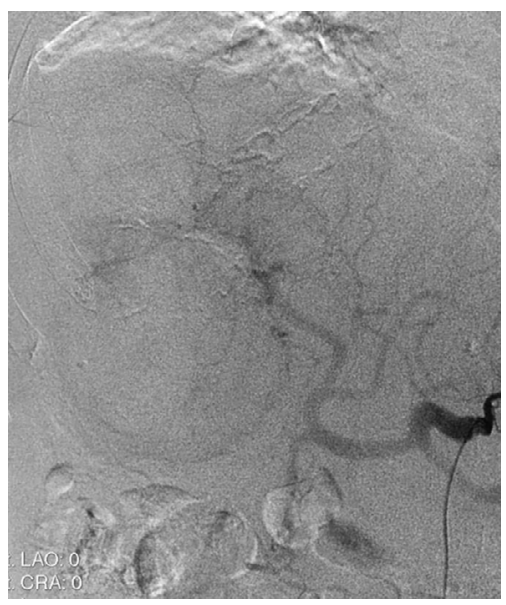

Figure 4. Imaging of the celiac and hepatic arteries showed a circumscribed non-vascular lesion with a combination of tumor and cystic features, although no clear extravasation was detected.

\section{Discussion}

Sarcomatoid HCC is a rare neoplasm of the liver. A previous study reported findings of sarcomatous changes in only $3.9 \%$ of $350 \mathrm{HCC}$ autopsies (2). The pathogenesis of sarcomatoid HCC has not been fully clarified. Sarcomatous components are thought to be derived from the processes of dedifferentiation or anaplasia in the setting of HCC, rather than the combination of the effects of HCC and sarcoma (2). Ishak and colleagues classified spindle cell (pseudosarcomatous or sarcomatoid)-type HCC as a HCC type in a working group sponsored by the World Health Organization (3). The tumor is characterized by a very poor prognosis as a result of rapid growth and metastasis at an early stage as well as low resectability and frequent recurrence after curative resection and even liver transplantation (1, 4-6). Hwang et al. reported 3 -year survival rates for sarcomatoid 

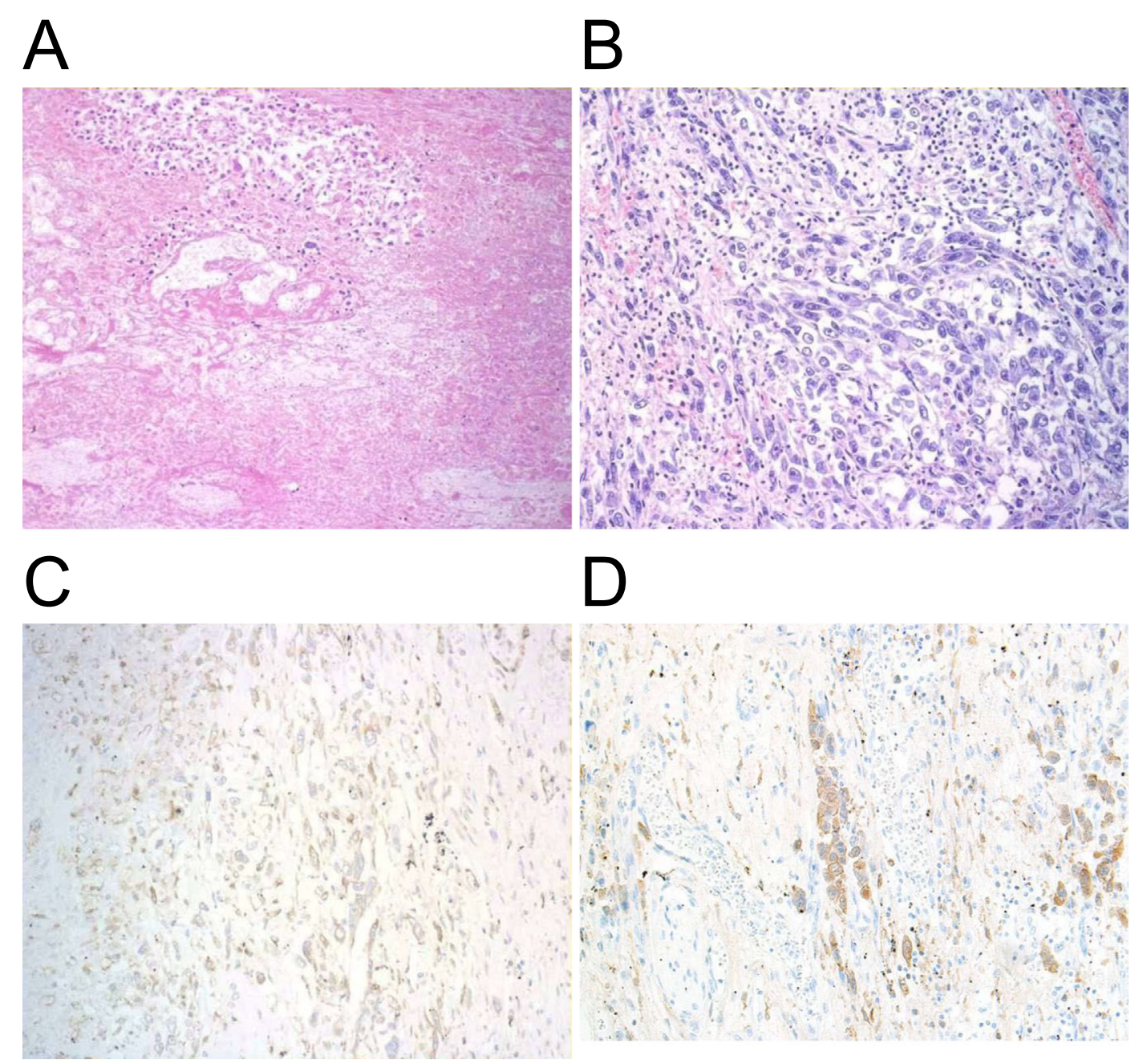

Figure 6. Microscopically, the tumor consisted of spindle-shaped tumor cells (A, B). An immunohistochemical examination revealed positive results for only vimentin (C) and hepatocytes (D).

HCC after liver resection and transplantation of $18.2 \%$ and $37.5 \%$, respectively (4). In the present case, the sarcomatoid changes were speculated to be induced by anti-HCC treatment, including radiofrequency ablation (RFA), percutaneous ethanol injection and transarterial chemoembolization, resulting in the degeneration, necrosis and regeneration of tumor cells $(7,8)$. However, previous studies have reported cases with no history of prior therapy $(8,9)$.

The CT findings of sarcomatoid HCC have been reported to include irregularly demarcated intrahepatic masses with delayed prolonged peripheral enhancement (10). However, no reports have indicated the features of sarcomatoid HCC on ultrasonography or CEUS. In the current case, we were able to observe the detailed appearance of sarcomatoid HCC on both ultrasonography and CEUS. Notably, the lesions, comprised of spindle-shaped tumor cells, were enhanced in the arterial phase and subsequently became hypo-enhanced starting at 30 seconds after contrast injection, while the peripheral area of the tumor remained enhanced and the necrotic lesions showed hyper-enhancement only along the periphery. Although this enhancement pattern on CEUS may represent metastasis or intrahepatic cholangiocarcinoma, the potential for sarcomatoid HCC should also be considered in cases involving these findings.

In the present case, the tumor appeared to have a cystic component and solid component based on the macroscopic findings, whereas CEUS and CT showed one cystic and two solid components; rapid progression of necrosis is considered to be the cause of this difference. According to the image inspection, the cells on one side of the solid component were still living, exhibiting contrast enhancement. However, at the autopsy, almost all of the cells were found to be necrotic. As a result, the tumor contained only one solid component based on the gross appearance. In fact, almost all of the tumor cells exhibited necrosis based on the microscopic findings. Therefore, we consider the cause of the necrosis to be the rapid growth of the tumor as well as the influence of transcatheter arterial embolization.

Sarcomatoid HCC appears to display a tendency to rapidly increase in size. However, no previous reports have described cases of sarcomatoid HCC rupture. In the present case, extravasation was confirmed on CEUS, and tumor rupture was diagnosed. Several recent reports have suggested that CEUS performed with Levovist (Schering, Berlin, Germany) or Sonazoid may also be useful for diagnosing ruptured HCC and identifying the bleeding site (11-13). Among 
10 patients with ruptured HCC who underwent CEUS with Levovist and abdominal angiography, Matsumoto et al. (9) found that CEUS was successfully used to differentiate active from inactive hemorrhage by detecting the contrast agent in ascites. In addition, Sugihara et al. (10) found that CEUS with Sonazoid is effective for identifying bleeding sites on the hepatic surface after RFA based on the presence of jet-like high-intensity echoes in pleural effusion, although the findings could not be confirmed on color Doppler imaging.

In conclusion, we encountered a case of ruptured sarcomatoid HCC. CEUS is potentially useful for reaching a diagnosis of sarcomatoid HCC.

The authors state that they have no Conflict of Interest (COI).

\section{References}

1. Tsujimoto M, Aozasa K, Nakajima Y, Kariya M. Hepatocellular carcinoma with sarcomatous proliferation showing an unusual and wide-spread metastasis. Acta Pathol Jpn 34: 839-845, 1984.

2. Kakizoe S, Kojiro M, Nakashima T. Hepatocelluar carcinoma with sarcomatous change: clinicopathologic and immunohistochemical studies of hepatocellular carcinoma chemical studies of 14 autopsy cases. Cancer 59: 310-316, 1987.

3. Anthony PP. Hepatocellular carcinoma: an overview. Histopathology 39: 109-118, 2001.

4. Hwang S, Lee SG, Lee YJ, et al. Prognostic impact of sarcomatous change of hepatocellular carcinoma in patients undergoing liver resection and liver transplantation. J Gastrointest Surg 12: 718-724, 2008.

5. Yamanaka J, Yamanaka N, Nakasho K, et al. Clinicopathologic analysis of stage II-III hepatocellular carcinoma showing early massive recurrence after liver resection. J Gastroenterol Hepatol 15: 1192-1198, 2000.

6. Eriguchi N, Aoyagi S, Okuda K, et al. Unusual liver carcinomas with sarcomatous features: analysis of four cases. Surg Today 31: 530-533, 2001.

7. Kojiro M, Sugihara S, Nakashima O, Kiyomatsu K. Hepatocellular carcinoma with sarcomatous change: a special reference to the relationship with anticancer therapy. Cancer Chemother Pharmacol 23: S4-S8, 1989.

8. Honda $\mathrm{H}$, Hayashi $\mathrm{T}$, Yoshida $\mathrm{K}$, et al. Hepatocellular carcinoma with sarcomatous change: characteristics findings of two-phased incremental CT. Abdom Imaging 21: 37-40, 1996.

9. Yokomizo J, Cho A, Yamamoto H, et al. Sarcomatous hepatocellular carcinoma without previous anticancer therapy. J Hepatobilary Pancreat Surg 14: 324-327, 2007.

10. Yoshida N, Midorikawa Y, Kajiwara T, et al. Hepatocellular carcinoma with sarcomatoid change without anticancer therapy. Case Rep Gastroenterol 7: 169-174, 2013.

11. Matsumoto N, Ogawa M, Nakagawara $H$, et al. Clinical efficacy of contrast-enhanced ultrasonography (CEUS) in the diagnosis of ruptured hepatocellular carcinoma (HCC). J Med Ultrasonics 34: 101-105, 2007.

12. Sugihara T, Koda M, Tokunaga $S$, et al. Contrast-enhanced ultrasonography revealed active thoracic bleeding. J Med Ultrasonics 37: 143-145, 2010.

13. Shiozawa $\mathrm{K}$, Watanabe M, Ikehara $\mathrm{T}$, et al. Usefulness of contrastenhanced ultrasonography in the diagnosis of ruptured hepatocellular carcinoma. Clin J Gastroenterol 6: 334-337, 2013.

(C) 2015 The Japanese Society of Internal Medicine http://www.naika.or.jp/imonline/index.html 